\title{
Predictores del desempeño en lectura y escritura de niños de primer grado ${ }^{1}$
}

\author{
Aldo Bazán R. ${ }^{2}$ \\ Instituto Tecnológico de Sonora
}

\author{
Beatriz Sánchez H. ${ }^{3}$ \\ Universidad de Sonora
}

\author{
Víctor Corral V. ${ }^{4}$ \\ Universidad de Sonora
}

\begin{abstract}
Se evaluó el desempeño de 288 niños de primer grado en tareas de lectura y escritura de diferente grado de complejidad. Se tomaron como predictores de los desempeños en las tareas evaluadas variables asociadas con la práctica docente y con antecedentes académicos inmediatos de tos niños, así como sus características físicas. Mediante la aplicación de un análisis de regresión múltiple se identificó que las variables de años de experiencia del maestro en primer grado, el juicio que emite respecto a la adquisición de la lectura y la escritura del niño, la presencia de padecimientos de tipo auditivo y/o visual en el niño, y el promedio logrado por los niños en los cursos de español al término del año escolar predicen aproximadamente un $40 \%$ del desempeño de los niños en todos los niveles de complejidad de las tareas de lectura y escritura evaluadas. Se discuten los resultados en términos de su implicación en la práctica docente. Palabras claves: lectura, escritura, desempeño escolar.
\end{abstract}

\section{Predictors of reading and writing achievement in first graders}

The achievement of reading and writing tasks of different complexity level was evaluated. Twohundred eighty-eight first-grade children responded to 76 items classified on the basis of one morphological and one functional criterion. Variables regarding teaching practices as well as children's characteristics were used as predictors of reading and writing tasks. A multiple regression analysis suggested that teacher's judgment concerning the child's reading-writing acquisition, some visual or auditive deficits, and final average on Spanish course explained almost $40 \%$ of the dependent variable variance. Results are discussed in terms of their teaching-leaming implications.

Key words: reading, writing, school achievement.

1 Esta investigación contó con el financiamiento del Consejo Nacional de Ciencia y Tecnología (CONACYT proyecto 29370-H) y del Programa de Apoyo a la lnvestigación del OTSON. Los autores agradecen a Claudia Tapia por su participación en la recolección y codificación de datos.

2 Magister en psicología por la UNAM-Iztacala y licenciado en la Universidad Nacional Federico Villarreal de Perú. Profesor-investigador asociado al ITSON México. Correo electrónico: aldo@itson.mx.

3 Es profesora en Ciencias de la Educación y en la maestría en Educación.

4 Doctor en psicología por la Universidad de Arizona. Es profesor titular e investigador nacional del Sistema Nacional de Investigadores de México. 

Leer y escribir son repertorios de conducta que en toda sociedad se consideran primordiales en el desarrollo del ser humano. Aprender a comportarse de manera efectiva en la lecto-escritura es indicador de haber alcanzado un nivel de desarrollo superior al logrado por alguien que sólo escucha y habla, además de que una vez que se alcanza el dominio en la lectura y la escritura se tiene la posibilidad de acceder a formas más complejas de desarrollo. No en balde los programas de enseñanza básica tienen como objetivo fundamental el que los niños aprendan a leer y escribir ya que tal aprendizaje si bien no garantiza, por lo menos probabiliza el que tengan acceso a mejores oportunidades de vida (CIDAC, 1991). En la mayor parte de los países latinos el leer y escribir son requisitos para cualquier oficio o trabajo por simple que éste sea (Ostrosky-Solis, 1988).

Sin embargo, existen indicadores de la existencia de dificultades para lograr este objetivo escolar. Según los reportes de índices de deserción y reprobación escolar, estas dificultades están asociadas, entre otros factores, a la falta de dominio en las habilidades lectoras y de escritura en los niños tanto a nivel mecánico como de comprensión (Ferreiro, 1990).

Los indicadores de fracaso escolar comunes son los siguientes: (a) índices de reprobación, (b) índices de deserción escolar y (c) bajos índices de eficiencia terminal. En el caso del estado de Sonora, en el ciclo escolar 1995-1996 el 6.3\% de los niños matriculados en los grados iniciales de educación básica no obtuvo notas aprobatorias; en todo el país, el índice de reprobación fue del $8.1 \%$. Esto significa que de cada 100 alumnos, a nivel nacional, 8 no acreditaron el año escolar mientras que en ele estado de sonora por cada 100 niños 6 no lo lograron pasar al siguiente año escolar. A nivel nacional durante este mismo periodo escolar el índice de deserción escolar fue del $3.0 \%$, mientras que en el estado de Sonora fue del $3.2 \%$; la eficiencia 
terminal lograda en el mismo periodo fue a nivel nacional del $65.9 \%$ mientas que en el estado de Sonora fue del 68.6\%; por cada 100 niños mexicanos que ingresaron a la primaria sólo 65 la concluyeron durante el periodo 1995-1996 (Secretaría de Educación y Cultura, 1997).

Gómez Palacio, López, González y Adame (1995) han señalado que los índices de reprobación y deserción están vinculados a las dificultades que los niños enfrentan en la materia de español; esta autora indica que el mayor porcentaje de deserción ocurre en los dos primeros años de educación primaria, grados que se consideran medulares pues es en ellos donde el niño adquiere las habilidades básicas para el manejo del sistema de escritura, así mismo señala que los índices de reprobación están más asociados a la enseñanza de la lectura y escritura que a la enseñanza de otras materias.

La problemática escolar ha salido de las aulas de clases para convertirse en un problema social que ha llamado la atención de organismos mundiales como la UNESCO (Bazán, 1999). La magnitud de la problemática ha dado lugar a que diversas disciplinas se den a la labor de generar conocimiento que permita subsanar y/o prevenir la incidencia de la misma; y que ha llevado a la creación de estrategias de alcance mundial para la prevención y/o corrección de los déficits identificados.

Dentro de su diversidad conceptual, la psicología como disciplina científica ha derivado una serie de investigaciones que han contribuido a la comprensión y explicación de los fenómenos involucrados en las situaciones de la enseñanza y el aprendizaje. A este respecto Trigo, Martínez y Moreno (1996) han señalado seis enfoques generales:

1. Enfoques que dirigen su atención a la importancia que tiene la organización y estructura de lo que se debe aprender (materia o contenidos), entre los que se pueden ubicar los trabajos derivados de la tecnología educativa (Cabero, 1989; Cruz, 1986). 
Predictores del desempeño en lectura y escritura de niños de primer grado

2. Enfoques que se centran en las características del individuo que aprende (alumno) en términos de las habilidades cognoscitivas y la personalidad. Entre los trabajos derivados de este se encuentran en su gran mayoría aquéllos que tienen como base la teoría psicogenética; estudios que enfatizan la participación de estructuras mentales y/o las habilidades cognoscitivas del educando (Castañeda, López, Orduña y Pineda, 1993; Castañeda y Orduña; 1996; Paris y Oka, 1980).

3. Enfoques que ponderan las características del que enseña como la clave en el proceso de enseñanza-aprendizaje, en términos de estilos de enseñanza (Diaz-Aguado, 1985).

4. Enfoques que señalan la importancia tanto de las habilidades y características del que aprende como las del que enseña. Estos estudios, originados del trabajo de análisis de estilos de crianza, plantean la contribución que las características -tanto del alumno como del profesor- tienen en el aprendizaje; sin embargo no analizan interacciones (Aubrey, 1994).

5. Enfoques que enfatizan la interacción alumno-maestro en el proceso de enseñanza aprendizaje, dentro de los cuales se pueden señalar como representativos los trabajos sobre la construcción de significados compartidos (Candela, 1993, 1994; Coll y Onrubia, 1993; Edwards y Mercer, 1988; Medina, 1980).

6. El enfoque que enfatiza la interacción alumno-profesor-materia como campo de relaciones funcionales. En este enfoque se encuentran los estudios generados a partir del modelo de campo (Kantor, 1959) y la concepción de la conducta ling, ística como conducta referencial (Kantor y Smith, 1975), así como la taxonomía funcional del comportamiento (Ribes y López, 1985).

Todos los enfoques han generado un cuerpo de conocimiento que puede aplicarse en la explicación de la adquisición y ejercicio de la lecto-escritura; sin embargo la presente investigación toma como base la perspectiva conductual, específicamente el sexto enfoque señalado por Trigo, Martínez y Moreno (1996), primeramente porque el modelo 
de campo propuesto por Kantor (1959) parte del supuesto de que el comportamiento psicológico es un fenómeno natural en términos del ajuste que los organismos hacen respecto a su medio ambiente, ajuste en el que no participan entidades de tipo trascendental. Además, la taxonomía funcional de la conducta propuesta por Ribes y López (1985) derivada del modelo de campo de Kantor, provee los lineamientos para caracterizar el comportamiento humano complejo desde una perspectiva conductual sin reducirlos a actos de corte mecánico.

El modelo de campo (Kantor, 1959) y la taxonomía funcional de la conducta (Ribes y López, 1985) tienen como supuestos fundamentales lo siguiente:

1. La conducta es una interacción organismo-ambiente, relación en la que participan como elementos los objetos del medio, las respuestas del organismo, un medio que posibilita el contacto entre ambos, y factores que pueden o no facilitar la ocurrencia del contacto entre los objetos del medio y la reactividad del organismo. Un segmento conductual (Kantor y Smith, 1975) puede representarse de la manera como se aprecia en la Figura 1

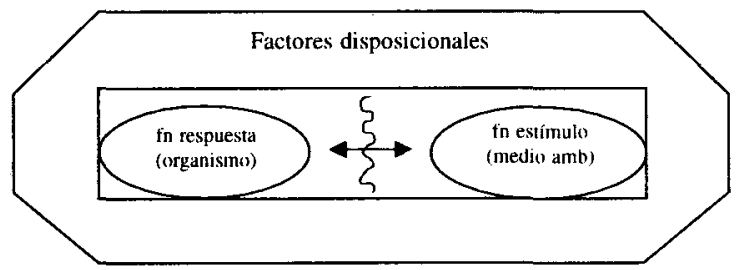

Figura 1. En un segmento conductual la función estímulo-respuesta interactúa con factores que pueden o no favorecerla.

2. La configuración particular que asuman los diversos elementos involucrados puede dar lugar a interacciones funcionalmente distintas; de este modo se tiene que una interacción en particular no 
Predictores del desempeño en lectura y escritura de niños de primer grado

se define por los elementos que participan en ella, sino en el arreglo que estos elementos tienen en un momento determinado.

Los contactos organismo-medio ambiente mediados por las características físico-químicas de los objetos de estimulación y de las respuestas del organismo son funcionalmente menos complejos que los contactos mediados por el lenguaje.

Los contactos organismo-medio ambiente que están apegados a las características situaciones concretas se identifican como contextuales, suplementarias y selectoras; las interacciones que están desligadas parcialmente de características situacionales concretas se identifican como sustitutivas referenciales, en tanto que las interacciones que no dependen de la situación concreta se identifican como sustitutivas no referenciales.

Con base en estos supuestos, las ejecuciones de los niños al leer y escribir pueden definirse como una conducta que puede caracterizarse en términos funcionales, es decir, como una relación que los niños establecen con las grafías. Caracterizar las ejecuciones de los niños en términos funcionales permite identificar el grado de complejidad en que los niños lee y escriben. Esto ofrece la ventaja de evaluar el dominio de la lectura y la escritura no sólo en términos de cuántas palabras lee y/o cuántos errores comete al escribir o leer (dominio mecánico), sino que además permite identificar el grado en que el niño mediante la lectura y la escritura puede o no entrar en contacto con situaciones pasadas o futuras, así como con atributos de los objetos o eventos. Adicionalmente permite identificar algunas variables que participan como facilitadoras del contacto entre la reactividad del niños y las características de la tarea (variables como factores disposicionales).

Diversas variables han sido señaladas como las determinantes de las ejecuciones de los niños en tareas de lectura y escritura. Por ejemplo, Mavrogenes y Bezrucczko (1993) señalan que el desarrollo 
de la lectura y la escritura está auspiciado por las actitudes y las expectativas que los niños, los padres y los maestros tienen respecto al aprendizaje de los niños. Smith (1990) observó que las actitudes hacia la lectura en la adolescencia predicen el ejercicio de la lectura en la vida adulta. Penny y Bond (1992) señalan que el nivel socioeconómico es una variable altamente predictiva del desempeño escolar de los niños porque implica el nivel educativo de los padres y las oportunidades que los niños tienen a materiales impresos, en tanto que la pertenencia étnica como variable no es susceptible de una operacionalización clara. Meyer, Wardrop, Hasting y Linn (1993) identifican como predictores de la lectura las habilidades desarrolladas en preescolar así como las condiciones de enseñanza bajo las cuales los niños aprender a leer en los primeros años de instrucción elemental.

El sexo de los niños también ha sido una variable a considerar. Por ejemplo, Smith (1990), Allred (1990) y Tiedemann y Faber, (1992) reportan que las niñas logran mejores puntajes en deletreo y comprensión de lectura que los niños.

Con base en lo anterior la investigación realizada tuvo dos objetivos fundamentales:

- Evaluar el dominio que los niños de primer grado tienen en tareas de lectura y escritura.

- Identificar algunas variables de tipo disposicional que explican la variabilidad de las respuestas de los niños en tareas de lectura y escritura. 


\section{Metodología}

\section{Participantes}

Participaron 10 grupos completos de primer grado de educación primaria pertenecientes a instituciones públicas de Cd. Obregón, Sonora con su respectiva maestra; 5 de los grupos pertenecían al sistema estatal y 5 al sistema federal. El total de los niños fue de 288 niños matriculados en el primer grado. 153 niños pertenecían al sistema estatal y 135 niños al sistema federal; 129 eran del sexo masculino y 159 del sexo femenino. Todos los niños estaban finalizando el primer grado de educación básica. Los diez grupos fueron proporcionados por las supervisiones locales de escuelas primarias de Cd. Obregón, con el consentimiento de los directores de escuelas y de las profesoras a cargo de los grupos.

\section{Instrumentos}

Para la evaluación de la lectura y la escritura se utilizó un instrumento que consiste en 76 reactivos que se clasifican con base en dos criterios: un criterio morfológico que se aplica para identificar una tarea de lectura o una tarea de escritura con base en la forma de la respuesta que se requiere en la tarea y, un criterio funcional que aplica para identificar el grado de complejidad de la tarea según el desligamiento de las características concretas de las tareas que se requiere para responder adecuadamente cada tarea. Para establecer el criterio funcional el instrumento de evaluación emplea los niveles descritos por la taxonomía funcional del comportamiento (Ribes y López, 1985). Los tipos de tareas empleados y su clasificación se sintetizan en el Cuadro 1. 


\section{Cuadro 1}

Evaluación funcional de la lectura y la escritura

\begin{tabular}{|c|c|}
\hline \multicolumn{2}{|l|}{ Evaluación } \\
\hline Descripción & Tareas implicadas \\
\hline $\begin{array}{l}\text { Nivel contextual } \\
\text { Tareas en donde el niño sólo tiene que ajustar sus respues- } \\
\text { tas al requerimiento de la tarea; el niño no puede alterar la } \\
\text { estructura de la tarea, solo puede entrar en contacto con } \\
\text { ella o no. }\end{array}$ & $\begin{array}{l}\text { - lectura de palabras }{ }^{1} \\
\text { - } \text { dictado de palabras }{ }^{2} \\
\text { - } \text { dictado de oraciones }^{2} \\
\text { - } \\
\text { escritura de nombres comunes a partir } \\
\text { de figuras }\end{array}$ \\
\hline $\begin{array}{l}\text { Nivel Suplementario } \\
\text { Tareas en donde el niño tiene que establecer una relación } \\
\text { entre estímulos modificando las características originales } \\
\text { de la tarea para responder a la relación formada por él }\end{array}$ & $\begin{array}{l}\text { - } \\
\text { completar dibujos y palabras } \\
\text { - completar dibujos y etiquetarlos } \\
\text { - } \\
\text { completar oraciones }\end{array}$ \\
\hline $\begin{array}{l}\text { Nivel Selector } \\
\text { Tareas en las que el responder del niño es condicional res- } \\
\text { pecto a las variaciones que se establecen entre los estímu- } \\
\text { los presentados; en este tipo de tareas la respuesta del niño } \\
\text { dependerá del establecimiento de relaciones entre estímu- } \\
\text { los de una manera particular mediada por un estímulo que } \\
\text { hace condicional la respuesta, mas que de los estímulos } \\
\text { per se }\end{array}$ & 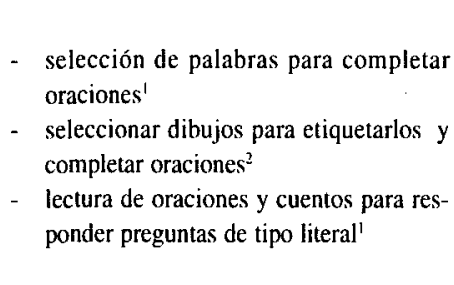 \\
\hline $\begin{array}{l}\text { Nivel Sustitutivo Referencial } \\
\text { Tareas en donde el responder del niño atiende a las pro- } \\
\text { piedades no aparentes y no presentes en tiempo y espacio } \\
\text { de los estímulos presentados, mediante la introducción de } \\
\text { circunstancias nuevas creadas a través del lenguaje. }\end{array}$ & $\begin{array}{l}\text { - lectura de oraciones y cuentos para res- } \\
\text { ponder a preguntas no literales' } \\
\text { - } \text { composición escrita a partir de dibujos en }^{\text {secuencia? }} \\
\text { - } \text { composición escrita a partir de una frase? }^{2} \text {. }\end{array}$ \\
\hline $\begin{array}{l}\text { Nivel Sustitutivo no referencial } \\
\text { Tareas donde el responder del niño está completamente } \\
\text { mediado por relaciones de orden ling,ístico; específica- } \\
\text { mente las tareas atienden al establecer relaciones entre } \\
\text { eventos y conceptos abstractos y/o a responder ante tales } \\
\text { relaciones }\end{array}$ & $\begin{array}{l}\text { - lectura de historia para opinar' } \\
\text { - lectura de dichos para interpretar' } \\
\text { - Definición de conceptos abstractos }\end{array}$ \\
\hline
\end{tabular}

Nota. 'tarea con morfología de respuesta de lectura.

?tarea con morfología de respuesta de escritura. 
La calificación de las respuestas de los niños a cada una de las tareas se hizo empleando una escala de 0 a 4 . La más alta puntuación posible en una tarea (4) indica que el niño responde adecuadamente a los requerimientos de la tarea mientras que la puntuación mínima (0) indica que la respuesta del niño no cumple de manera alguna el criterio de logro requerido por la tarea; respuestas intermedias se ubican en los puntajes de 1, 2 y 3 según las características de la respuesta (para detalles de la calificación consultar Rojas y Zavala, 2000).

La validez y confiabilización del instrumento empleado se obtuvo a través de un análisis factorial confirmatorio mediante la aplicación de la estrategia multicaracterísticañmultimétodo (Campbell y Fiske, 1959; Widaman, 1985), a partir de la cual se determinó que cada una de las tareas recibió simultáneamente pesos factoriales del correspondiente factor morfología y del correspondiente factor nivel funcional. Asimismo se determinó que las tareas preescritas como pertenecientes al mismo nivel de complejidad presentaron convergencia en el factor funcional correspondiente (validez convergente) al tiempo que presentaron divergencia con las tareas que se prescribieron como pertenecientes a niveles de complejidad extremos (validez divergente), pero no contiguos. Respecto al factor morfología se determinó que no había divergencia significativa.

Para recolectar la información respecto a las variables predictoras se utilizó un formato de ficha de identificación a ser llenada por cada una de las maestras de los niños participantes. En esta ficha se le solicitó a cada maestra que diera información respecto a las siguientes variables:

\section{Alumno}

- Presencia de problemas físicos tales como visual (uso de lentes), auditivo (uso de aparato auditivo) y/o de articulación diagnosticado.

- $\quad$ Sistema de preescolar (público o privado).

- Veces que ha cursado el primer grado de primaria.

- Promedio final en español. 
Aldo Bazán R., Beatriz Sánchez H. y Víctor Corral V.

- Promedio final en matemáticas.

- Promedio final general obtenido al finalizar el año escolar.

- Juicio del maestro acerca de la adquisición de la lectura y la escritura.

\section{Docencia}

- Sistema escolar al que pertenece la institución en la que labora.

- Años que tiene impartiendo primer grado.

- Estrategia que utilizó en el año escolar 98-99 para la enseñanza de la escritura y la lectura (silábico, fonético, global, PRONALES, o la combinación de dos o más de las estrategias señaladas).

\section{Procedimiento}

La aplicación de la evaluación se realizó en una aula de clases con el mobiliario propio, proporcionada por cada una de las escuelas participantes ( 10 escuelas; 5 estatales y 5 federales).

La aplicación de la evaluación de la lectura y la escritura se aplicó de manera individual a cada niño según la prescripción del instrumento (para detalles consúltese a Zavala y Rojas, 1999). A cada maestra participante se le brindó una breve explicación de la investigación; se le dio a cada una la ficha de identificación para que proporcionara la información requerida aclarándole que la misma sería confidencial y únicamente utilizada para los fines de la investigación.

Una vez aplicada la evaluación a los niños participantes y obtenida la información de las maestras se agradeció a todos su participación.

\section{Resultados}

En cuanto a la evaluación de lectura y escritura, las respuestas de los niños en cada una de las tareas de lectura y escritura se calificaron en una escala de 0 a 4 . Con base en esta escala se obtuvieron los 
promedios por tipo de tarea (nivel funcional), que se presentan en la Figura 2.

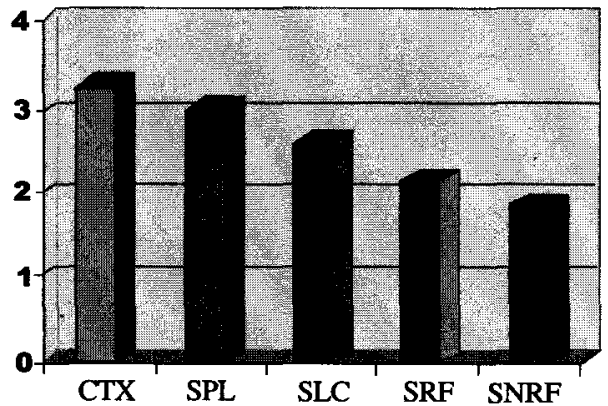

Figura 2. Se presenta el puntaje promedio obtenido por los 288 niños de primer grado evaluados en tareas de lectura y escritura de diferente complejidad.

En esta Figura puede observarse que en todos los niveles se obtuvieron puntajes, sin embargo se advierte que es las tareas mas simples, primeros dos niveles, los niños alcanzaron puntajes por encima de 2.5 y que a medida que responden ante tareas de mayor complejidad, los puntajes promedio van decrementando. Un vistazo a las desviaciones estándar identificadas en cada nivel de complejidad de tareas (Cuadro 1) señala que a medida que los niños responden a niveles mas complejos, la variabilidad de sus respuestas se va incrementando.

Este decremento progresivo de las medias y aumento de la desviación estándar indica que los desempeño de los niños pueden clasificarse en términos de su complejidad. El instrumento utilizado parte del supuesto de que las tareas que implican únicamente decodificación son menos complejas que aquellas que implican interpretación; los datos apoyan el supuesto (Cuadro 2). 


\section{Cuadro 2}

Medias y desviaciones estándar obtenidas en cada serie de tareas

\begin{tabular}{|l|llc|}
\hline \multicolumn{1}{|c}{ Escala } & $x$ & $\sigma$ & Alfa Cronbach \\
\hline - Lectura palabras. & 3.58 & 0.89 & 0.91 \\
- Dictado palabras & 3.47 & 0.85 & 0.89 \\
- Dictado oraciones. & 3.52 & 1.08 & 0.86 \\
- Escritura nombres & & & \\
comunes. & 3.26 & 0.79 & 0.88 \\
\hline - Completar figuras y nombres. & 3.48 & .0 .89 & 0.86 \\
- Completar figuras y etiquetarlas & 3.22 & 0.86 & 0.86 \\
- Completar oraciones & 2.26 & 0.91 & 0.91 \\
\hline - Seleccionar palabras & 2.85 & 1.27 & 0.90 \\
- Seleccionar figuras para & & & \\
etiquetarlas & 3.01 & 1.22 & 0.95 \\
- Preguntas literales oraciones & 2.33 & 1.37 & 0.93 \\
- Preguntas literales cuentos & 2.36 & 1.51 & 0.94 \\
\hline - Preguntas no literales & & & \\
oraciones & 2.23 & 1.35 & 0.92 \\
- Preguntas no literales cuentos & 2.49 & 1.61 & 0.95 \\
- Composición escrita por & & & \\
dibujos & 1.97 & 1.23 & 0.81 \\
- Composición escrita por & & & \\
instrucción escrita & 1.70 & 1.10 & 0.79 \\
\hline - Lectura para opinar & 1.71 & 1.21 & 0.96 \\
- Lectura de dichos & 1.50 & 1.11 & 0.91 \\
- Escritura tema simbólico & 2.11 & 1.31 & 0.92 \\
\hline
\end{tabular}

Nota. El coeficiente Alfa indica consistencia interna para cada escala.

En relación a la práctica docente, por procedimiento de selección de los grupos para la evaluación de la lectura y la escritura, 5 maestras pertenecían al sistema estatal y 5 al sistema federal evaluación. De acuerdo al reporte de las maestras respecto a los años que tienen impartiendo primer grado se identificó que las 5 maestras que pertenecen al sistema estatal tienen un promedio de 4 años consecutivos, mientras que las 5 maestras pertenecientes al sistema federal tienen 
un promedio de 2 años. Esta diferencia de medias es significativa ( $t$ $=4.73, p=.0001$ ).

Todas las maestras participantes maestras reportaron que durante el año escolar utilizaron mas de una estrategia para la enseñanza de la lectura; no se identificó apego a alguna estrategia en particular. (Silábico, Fonético, Global, PRONALES).

En relación a los niños, se identificó que de los 288 niños evaluados el $11.4 \%$ presentaba algún tipo de problema auditivo, visual y/ o de articulación. De este $11.4 \%$, el $46 \%$ presentaba problemas de tipo visual (uso de lentes); el $3 \%$ presentaba problemas de tipo auditivo; el $39 \%$ fue reportado con problemas de articulación (diagnosticado), mientras que un $12 \%$ fue reportado por presentar la combinación de estos tipos de deficiencias.

El 86\% de los niños asistió al preescolar público, mientras que el $14 \%$ restante asistió a preescolar privado. No hay diferencias significativas en relación al sexo. El $97 \%$ de los niños evaluados estaba cursando por primera vez el primer grado, el 3\% restante lo estaba cursando por segunda vez. No hay diferencias significativas en relación al sexo, ni al sistema escolar.

El promedio logrado al finalizar el ciclo escolar en la materia de español fue para los niños inscritos en el sistema estatal, de 8.45; y para los niños del sistema federal, de 8.32; no hay diferencias significativas entre los promedios. Al igual que en la materia de español, en matemáticas no se identificaron diferencias significativas entre los promedios obtenidos por los niños pertenecientes al sistema estatal y el sistema federal: 8.56 y 8.28 , respectivamente. Respecto al promedio general obtenido por los niños en el ciclo escolar 98-99, los niños del sistema estatal obtuvieron un promedio de 8.93, en tanto que los niños pertenecientes al sistema federal, un promedio de 8.6. La diferencia fue significativa $(t=2.74, p=.0065)$. 
Según el juicio de las maestras, de los 288 niños evaluados, el 92\% sí aprendió a leer y a escribir y el $8 \%$ restante no lo logró. No se identificó relación alguna entre esta variable y el sistema escolar al que pertenece el niño, ni tampoco en relación a la estrategia de enseñanza que reportaron utilizar las maestras.

Por otro lado, los predictores de las ejecuciones de lectura y escritura se analizaron mediante el análisis de regresión múltiple. Se identificó que de las diez variables consideradas, las que se relacionaron de manera significativa con las ejecuciones de los niños en los cinco tipos de tareas de lectura y escritura fueron las siguientes: (a) la presencia de problemas de tipo visual, auditivo y/o de articulación; (b) Los años de experiencia de las profesoras, (c) los promedios obtenidos al final de ciclo escolar en la materia de español; d) el juicio de la maestra respecto a la adquisición de la lectura y la escritura, y e) la asistencia al preescolar sólo para las ejecuciones de las tareas correspondientes al tercer nivel de complejidad (nivel selector). Los valores de la relación de estas variables con las ejecuciones de los niños en cada uno de los cinco tipos de tareas de lectura-escritura se presentan en el Cuadro 3.

Como puede identificarse en el Cuadro 3, las cuatro variables están explicando más del $40 \%$ de la variabilidad de las respuestas de los niños en las tareas de lectura y escritura en cada uno de los niveles funcionales $(R \leq)$. En el caso de los repertorios selectores se identifica que la variable asistencia al preescolar contribuye a la explicación, lo cual es comprensible puesto que es en el preescolar en donde los niños ejercitan habilidades que implican la clasificación y agrupamiento arbitrario de objetos y eventos. 
Predictores del desempeño en lectura y escritura de niños de primer grado

\section{Cuadro 3}

Valores de R $\leq$ para cada una de las Escalas de la Evaluación Funcional de la Lectura y la Escritura

\begin{tabular}{|c|c|c|c|c|c|c|}
\hline \multirow[b]{2}{*}{ Predictores } & \multicolumn{3}{|c|}{ Escala Contextual } & \multicolumn{3}{|c|}{ Escala Suplementaria } \\
\hline & C. reg. & $t$ & $p<.05$ & C. reg. & $t$ & $p<.05$ \\
\hline Experiencia docente & 0.043 & 3.68 & .0003 & 0.053 & 4.01 & .0001 \\
\hline Problemas físicos & -0.121 & -3.30 & .0011 & -0.143 & -3.51 & .0005 \\
\hline Promedio español & 0.256 & 4.64 & .0001 & 0.264 & 4.31 & .0001 \\
\hline Juicio de la maestra & -1.173 & -9.39 & .0001 & -1.289 & -9.28 & .0001 \\
\hline Valor de $\mathbf{R}^{2}$ & \multicolumn{3}{|c|}{0.6650} & \multicolumn{3}{|c|}{0.6605} \\
\hline \multirow[t]{2}{*}{ Predictores } & \multicolumn{3}{|c|}{ Escala Selectora } & \multicolumn{3}{|c|}{ Escala Sustitutiva Referencial } \\
\hline & C. reg. & $t$ & $p<.05$ & C. reg. & $t$ & $p<.05$ \\
\hline Experiencia docente & 0.071 & 3.58 & .0001 & 0.068 & 3.22 & .0014 \\
\hline Problemas físicos & -0.214 & -3.48 & .0006 & -0.224 & -3.45 & .0006 \\
\hline Promedio español & 0.538 & 5.81 & .0001 & 0.563 & 5.75 & .0001 \\
\hline Juicio de la maestra & -1.169 & -5.58 & .0001 & -0.654 & -2.95 & .0034 \\
\hline Asistencia a preescolar & 0.936 & 2.07 & .0390 & & & \\
\hline Valor de $\mathbf{R} \leq$ & & 0.59 & & & 0.539 & \\
\hline \multirow[t]{2}{*}{ Predictores } & \multicolumn{3}{|c|}{$\begin{array}{l}\text { Escala Sustitutiva } \\
\text { no Referencial }\end{array}$} & & & \\
\hline & C. reg. & $t$ & $p<.05$ & & & \\
\hline Experiencia docente & 0.076 & 3.67 & .0003 & & & \\
\hline Problemas físicos & -0.242 & -3.76 & .0002 & & & \\
\hline Promedio español & 0.442 & 4.56 & .0001 & & & \\
\hline Juicio de la maestra & -6.664 & -3.02 & .0027 & & & \\
\hline Valor de $\mathbf{R} \leq$ & & & 0.4447 & & & \\
\hline
\end{tabular}

\section{Conclusiones}

Con base en los resultados descritos puede señalarse que las ejecuciones de los niños en tareas de lectura y escritura pueden caracterizarse en términos de su complejidad funcional, entendida ésta en términos del tipo de mediación involucrada en la interacción niñoñgrafía. Las expectativas de los investigadores respecto al desempeño de los niños fueron superadas por lo encontrado; antes de la aplicación de la 
evaluación se creyó que los niños -que se encontraban culminando el primer grado escolar- cumplirían sin dificultades los criterios de ejecución de las tareas correspondientes a los tres primeros niveles, por ser tareas que demandan un tipo de mediación situacional, y escasamente llegarían con éxito a las tareas mas complejas. Sin embargo, llama la atención de que si bien es cierto que el promedio de ejecución en las tareas complejas es inferior al obtenido en las tareas simples, dichos promedios se encuentran cercanos a la mediana de la escala de calificación empleada; esto es, que para ser niños que recién están en proceso de adquisición de la lectura, su desempeño es alto.

Lo anterior puede tener relevancia para la práctica docente en el primer grado. Si los niños evaluados lograron realizar tareas simples y complejas, y tal desempeño no tiene relación, al menos en la muestra de niños empleada, con las estrategias empleadas por las maestras, es necesario considerar otras variables. Es común que los padres de familia tiendan a creer que los logros o fracasos de sus hijos se deban a una mala enseñanza o a problemas propios a los niños. Sin embargo los datos aquí presentados sugieren que no es posible atribuir el éxito a un solo factor. Por ejemplo, en esta investigación se identificó que los años que un profesor tiene frente a grupo en un mismo grado escolar pueden predecir el desempeño de los niños pero que también la presencia de problemas de tipo sensorial y/o articulatorio contribuyen.

Es cierto que las variables empleadas en esta investigación son mínimas comparadas con las cotidianamente ocurren en el contexto de la enseñanza y el aprendizaje; piénsese por ejemplo en todos aquellos factores de tipo familiar e institucional que están implicados. Identificar las causas de los desempeños académicos de los niños implica sistematicidad y compromiso de los involucrados en la práctica docente. 
Predictores del desempeño en lectura y escritura de niños de primer grado

\section{Referencias}

Allred, R. (1990). Gender differences in spelling achievement in grades 1 through 6. Journal of Educational Research. 83, 187-193.

Aubrey, C. (1994). An investigation of children's knowledge of mathematics at school entry and the knowledge their teacher hold about teaching and learning mathematics young learners and mathematics subjet knowledge. British Educational Research Journal, 20, 1, 105-115.

Bazán, A. (1999). La enseñanza de la lectura y la escritura en la escuela primaria pública. En A. Bazán (Comp), Aportes Conceptuales y Metodológicos en Psicología Aplicada. Cd. Obregón: ITSON.

Cabero, J. (1989). Tecnología Educativa: utilización didáctica del video. Barcelona: PPU.

Candela A. (1993). La construcción discursiva de la ciencia en el aula. Investigación en el aula, 21, 31-37.

Candela A. (1994). La enseñanza de la ciencia y el análisis del discurso. En M. Rueda, B. Delgado y Z. Jacobo (Eds.), La etnografía en educación. México: UNAM-CISE.

Castañeda, S., López, M., Orduña, J. y Pineda, L. (1993). Un marco de trabajo experimental y neurocomputacional para el estudio de la estructuración del conocimiento a partir de lo leído. Revista Latina de Pensamiento y Lenguaje, 1, 2, 201-232.

Castañeda, S. y Orduña, J. (1996). Investigación cognitiva en aprendizaje y enseñanza de ciencias, artes y técnicas. Revista Latina de Pensamiento y Lenguaje, 4, 1, 69-94.

Centro de Investigación y Desarrollo. (1991). Educación para una economía competitiva. México: CIDAC.

Coll, C. y Onrubia, J. (1993). El análisis del discurso y la construcción de significados compartidos en el aula. Revista Latina de Pensamiento y Lenguaje, 1, 2, 241-260.

Cruz, J. (1986). Teorías del aprendizaje y tecnología de la enseñanza. México: Trillas.

Diaz-Aguado, M. (1985). Estilos de enseñanza. En J. Beltrán (Ed.), Psicologia de la Educación. Madrid: UNED. 
Edwards, D. y Mercer, N. (1988). El conocimiento compartido: El desarrollo de la comprensión en el aula. Barcelona: Paidós.

Ferreiro, E. (1990). Alternativas a la comprensión del analfabetismo en la región. Tarea, 24, 5-16.

Gomez, P. M., López, M., González, L. y Adame, M. (1995). La lectura en la escuela. México: SEP.

Kantor, J. (1959). Interbehavior psychology. Chicago: Principia Press.

Kantor, J. y Smith, N. (1975). The science of psychology: an interbehavioral survey (Cap.3). Chicago: Principia Press.

Medina, A. (1980). Didáctica e interacción en el aula. Madrid: Cincel.

Ostroski-Solis, (1988). Características de la lectura y tipos de lectores en una población escolar mexicana. En A. Ardila y F. Ostrosky-Solis (Eds.), Lenguaje oral y escrito. México: Trillas.

Paris, S. y Oka, E. (1980) Children's reading strategies, metacognition, and motivation. Development Review, 6, 25-31.

Ribes, E. y López, F. (1985). Teoría de la conducta: un análisis de campo y paramétrico. México: Trillas.

Rojas, G. y Zavala. M. (2000). Validez y confiabilidad de un instrumento de evaluación funcional de la lectura y la escritura en niños de primer grado. Tesis licenciatura. Instituto Tecnológico de Sonora, Cd. Obregón.

Secretaría de Educación y Cultura (SEC) (1997). Infancia y adolescencia. Cuaderno Estadístico. Hermosillo: autor.

Trigo, E., Martínes, R. y Moreno, R. (1996). Niveles funcionales en la comunicación educativa. En L. Amador (Comp.), La psicología hoy: algunos campos de actuación. Sevilla: UNED Centro Asociado.

Widaman, K. (1985). Hierarchically nested covariance structure models for multitrait-multimethod data. Applied Psychological Measurement, 9, $1-26$. 